\title{
Identification of Type Ia Supernovae at Redshift 1.3 and Beyond with the Advanced Camera for Surveys on HST $^{1}$
}

\author{
Accepted for Publication by the Astrophysical Journal Letters
}

\begin{abstract}
Adam G. Riess ${ }^{2}$, Louis-Gregory Strolger ${ }^{2}$, John Tonry ${ }^{3}$, Zlatan Tsvetanov ${ }^{2}$, Stefano Casertano $^{2}$, Henry C. Ferguson ${ }^{2}$, Bahram Mobasher ${ }^{2}$, Peter Challis ${ }^{4}$, Nino Panagia ${ }^{2}$, Alexei V. Filippenko ${ }^{5}$, Weidong $\mathrm{Li}^{5}$, Ryan Chornock ${ }^{5}$, Robert P. Kirshner ${ }^{4}$, Bruno Leibundgut ${ }^{6}$, Mark Dickinson $^{2}$, Anton Koekemoer ${ }^{2}$, Norman A. Grogin ${ }^{2}$, and Mauro Giavalisco ${ }^{2}$
\end{abstract}

\begin{abstract}
We present discoveries of SNe Ia at $z>1$ and the photometric diagnostic used to discriminate them from other types of SNe detected during the GOODS Hubble Space Telescope Treasury program with the Advanced Camera for Surveys (ACS). Photometric redshift measurements of the hosts combined with deep f606w, f775w, and f850lp imaging discriminates hydrogen-rich SNe II from SNe I at $z>1$ by exploiting the ultraviolet (UV) deficit in the energy distributions of SNe Ia. This sorting allows rapid follow-up of space-based discoveries. Subsequent spectroscopy of 11 GOODS SNe Ia obtained from the ground and with the grism on ACS confirmed the reliability of our photometric screening. We present the highest-redshift spectrum of any supernova published to date, SN Ia $2002 \mathrm{fw}$ at $z=1.3$ observed near maximum brightness with the ACS grism. The lack of UV flux for true SNe Ia provides an effective tool for our ongoing efforts to build a sample of SNe Ia at $1.2<z<1.8$ which will extend the useful range of the magnitude-redshift relation of SNe Ia.
\end{abstract}

subject headings: supernovae: general — cosmology: observations

\footnotetext{
${ }^{1}$ Based on observations with the NASA/ESA Hubble Space Telescope, obtained at the Space Telescope Science Institute, which is operated by AURA, Inc., under NASA contract NAS 5-26555

${ }^{2}$ Space Telescope Science Institute, 3700 San Martin Drive, Baltimore, MD 21218

${ }^{3}$ Institute for Astronomy, University of Hawaii, 2680 Woodlawn Drive, Honolulu, HI 96822

${ }^{4}$ Harvard-Smithsonian Center for Astrophysics, 60 Garden St., Cambridge, MA 02138

${ }^{5}$ Department of Astronomy, 601 Campbell Hall, University of California, Berkeley, CA 94720-3411

${ }^{6}$ European Southern Observatory, Karl-Schwarzschild-Strasse 2, Garching, D-85748, Germany
} 


\section{Introduction}

The unexpected faintness of Type Ia supernovae (SNe Ia) at $z \approx 0.5$ provides direct evidence that the expansion of the Universe is accelerating, propelled by "dark energy" (Riess et al. 1998; Perlmutter et al. 1999). Augmented samples of SNe Ia extended to $z \approx 1$ (Tonry et al. 2003; Suntzeff et al. 2003; Leibundgut et al. 2003; Blakeslee et al. 2003), as well as more detailed studies of SNe Ia (see Leibundgut 2001 and Riess 2000 for reviews), have strengthened the case for the existence of dark energy based on supernovae. The new cosmic concordance model contains both dark matter and dark energy (i.e., $\Omega_{M} \approx 0.3$ and $\left.\Omega_{\Lambda} \approx 0.7\right)$ and is sharply delineated by the convergence of three independent probes of the $\Omega_{M}-\Omega_{\Lambda}$ plane: the cosmic microwave background (CMB), SNe Ia at $z<1$, and large-scale structure (LSS). However, when the number of constraints nearly equals the number of parameters little power remains to test the validity of the paradigm. Efforts to use the same measurements to fit an additional parameter, such the equation of state for dark energy, are vulnerable to systematic errors without redundant measurements.

A simple prediction of the new cosmological model is the weakening of dark energy relative to dark matter in proportion to $(1+z)^{3}$, leading to an earlier epoch dominated by dark matter, with decelerating expansion. If we fail to see deceleration, the evidence for dark energy is in doubt. The tools for this test are SNe Ia at $z>1$, whose leverage to measure past deceleration grows with redshift. SNe Ia at $z>1$ also create the opportunity to amplify the effects of any astrophysical contaminants such as chemistry, age, or strange dust that might contaminate the dark energy signal from SNe Ia.

To date, the reach of discovery from the ground has been limited to $z<1.2$ (Aldering et al. 1998; Coil et al. 2000; Tonry et al. 1999, 2003). Pursuit of SNe Ia to higher redshifts is inhibited by the bright night sky redward of $\sim 8000 \AA$. Searches at bluer wavelengths are starved of photons by the intrinsic deficiency of SNe Ia in the UV. Even at $1.0<z<1.2$, detections of SNe Ia from the ground are often not reliable. Success requires unusually favorable conditions and daringly low significance thresholds for detections, but attempts can result in false discoveries, inconclusive spectra, or incomplete photometric records (e.g., SN 1999fo, SN 1999fu and SN 1999fv; Tonry et al. 2003). Spectroscopic screening of low-confidence SN Ia candidates at $z>1$ is always time-consuming and often impractical and unsuccessful.

From space these challenges are largely mitigated. Gilliland \& Phillips (1998) reported the detection of SN $1997 \mathrm{ff}$ by WFPC2 on the Hubble Space Telescope (HST). Using serendipitous follow-up of the field by NICMOS, Riess et al. (2001) showed that this SN was almost certainly of type Ia at $z \approx 1.7$ and its apparent magnitude consistent with deceleration. However, a single, sparsely observed SN is insufficient to guard against a 
spurious conclusion caused by lensing (Benítez et al. 2002) or an unexpected SN type.

An unprecedented opportunity to collect a significant sample of SNe Ia at $1<z<2$ occurred in the eleventh year of HST owing to three factors: the installation of a wider and faster ACS (Ford et al. 2003), the refurbishment of NICMOS, and the periodic (45 day interval) multi-color imaging of two $10^{\prime} \times 15^{\prime}$ fields with ACS by the GOODS Treasury Program (Giavalisco et al. 2003). Each of the 8 repeated epochs of the GOODS imaging was expected to yield 1-2 SNe Ia at $z>1.2$ as well as 3-5 other SNe. The ability to discriminate the SNe Ia at $z>1.2$ from other transients with high confidence is essential to avoid following the wrong objects, or squandering spectroscopic time and to permit prompt HST monitoring via a pre-allocated program . The combined GOODS and SN "piggyback" program (GO 9352; Riess, PI) constitutes the first program designed to discover and follow SNe from space.

We have used observed colors and photometric redshifts to identify likely SNe Ia at $z>1$ via their intrinsic UV deficit. Subsequent measurements using the grism on ACS have provided the spectroscopic confirmation at redshifts unreachable from the ground including our first example and the highest redshift supernova yet measured, in this case a SN Ia at $z=1.3$. Here we report on a novel photometric method used to identify candidate SNe Ia at $z>1$ and demonstrate its spectroscopic confirmation. Elsewhere we report details of the search for transients and their rates (Strolger et al. 2003; Dahlen et al. 2003c), as well as the constraints on cosmology and the nature of distant SNe Ia (Riess et al. 2003).

\section{Identifying SNe Ia at $z>1$; the UV Deficit}

The modern classification scheme of SNe includes at least 7 primary subtypes (SN IIL, IIP, IIb, IIn, Ia, Ib, and Ic) identified via the presence or absence of key features in their optical spectra (see Filippenko 1997 for a review). The major dichotomy of classification, separates SNe with and without hydrogen (SNe II and I, respectively) in their spectra. Unfortunately, this historical convention fails to reflect current understanding which recognizes a different, more physical bifurcation, that of explosion mechanism. SNe Ia are believed to arise from the thermonuclear disruption of a white dwarf and all other subtypes via core collapse in a massive $\left(z 10 M_{\odot}\right)$ star with varying degrees of mass loss (Wheeler \& Harkness 1990; Filippenko 1997).

Explosive thermonuclear burning of the degenerate SN Ia progenitor produces $0.3-1$ $M_{\odot}$ of iron-group metals. Owing to its high velocities and great abundance, Fe II resonantly scatters much of the thermal continuum flux in the UV $(\lambda<3300 \AA)$, imprinting 
a characteristic "UV deficit" in the spectral energy distributions of SNe Ia. In contrast, the most common form of core-collapse SNe, those with hydrogen-rich, electron-scattering atmospheres, are strong emitters in the UV. As shown in Figure 1, the UV deficit of SNe Ia provides a useful, photometric method to discriminate between the two most frequently observed subtypes of SN, SNe Ia and SNe II. Rest-frame optical $(\lambda>3300 \AA)$ color discrimination of SNe has been shown by Poznanski et al. (2002) to be of use for this purpose, though photometric differences are more subtle at these wavelengths than in the UV.

Although SNe Ia may be routinely discovered and identified via photometric means, misidentification is possible. Core-collapse SNe with negligible hydrogen (SNe Ib and Ic) also have weak UV flux. However, such SNe are less common in the field than hydrogen-rich core-collapse SNe by a factor of $\sim 3$ (Cappellaro et al. 1997; Cappellaro, Evans, \& Turatto 1999; W. Li and A. V. Filippenko, 2003, private communication), and most are fainter than SNe Ia by 1-3 mag, effectively reducing their discovery rate in a magnitude-limited survey (e.g., Li et al. 2001). More troublesome is a subset of SNe Ic which attain optical luminosities comparable to those of SNe Ia and would be the chief contaminant to SNe Ia identified in magnitude-limited surveys in the optical. However, past surveys of this nature demonstrate that the degree of contamination is small. Ultra-luminous SNe Ic constitute only a few percent of SNe found by the Calán-Tololo Survey (Hamuy et al. 1996; Clocchiatti et al. 2000), the Abell Cluster Search (Germany et al. 2003), the Lick Observatory Supernova Search (Filippenko et al. 2001; W. Li and A. V. Filippenko, 2003, private communication), and the Nearby SN Factory Search (P. Nugent, 2003, private communication), or in compilations of past surveys (Richardson et al. 2002). Heavily reddened core-collapse SNe could mimic the colors of a SN Ia in the UV, but the total absorption would severely reduce their detection rate at $z>1$. Without a significant evolution in the relative rates of SN types or their photometric properties, the expected frequency of misidentification of candidate SN Ia targeted by this diagnostic would be less 1 in 10, although the rate may rise at higher redshifts. Because cosmological measurements from SNe Ia are statistical in nature, modeling of the frequency of contamination can be used to correct a sample but the best defense against sample contamination is spectroscopic screening.

The GOODS Survey provides the means to use this photometric discriminant for SNe Ia at $z>1$. SNe in the GOODS survey were discovered by image subtraction in $2000 \mathrm{~s}$ exposures in ACS $f 850 l p$, a passband that includes the rest-frame optical ( $U B V R I$ ) up to $z \approx 1.5$ and allows for the detection of SNe Ia up to $z \approx 1.8$. Simultaneous imaging in $f 775 \mathrm{~W}$ and $f 606 \mathrm{w}$ provides a useful measurement of the UV flux at $z>1$. As shown in Figure 2, the expected $f 775 w-f 850 l p$ and $f 606 w-f 850 l p$ colors of SNe Ia and SNe II 
diverge at $z>1.0$ and $z>0.7$, respectively, with apparent differences reaching 1-3 mag. SNe Ia at $z>1.3$ are expected to be so faint in $f 606 w$, that such SNe should appear as "drop-outs." The survey limit of $f 850 l p \approx 25.8 \mathrm{mag}$ (see Strolger et al. 2003 for more precise values) restricts the discovery of SNe at $z>1.2$ to $M_{B}<-17.5$ mag and at $z>1.5$ to $M_{B}<-18.5 \mathrm{mag}$.

Within 6 to 18 hours after a repeat visit of the Chandra Deep Field South or Hubble Deep Field North, each GOODS epoch was differenced with the preceding epoch to identify SNe. Follow-up spectroscopy of most candidates with $f 850 l p<24$ mag was attempted from the ground with the Keck and Magellan telescopes during the next new moon and are reported elsewhere (Riess et al. 2003). Candidates whose host photometric redshift indicated a high likelihood of $z>1.2$ and whose colors indicated a good match to the UV deficit of an SN Ia at the host redshift were targeted for follow-up with HST using ACS direct imaging, NICMOS, and in some cases ACS grism spectroscopy. All likely SNe were reported in the IAU Circulars within 72 hours of discovery, 43 to date (Giavalisco et al. 2002; Riess et al. 2002, 2003; Strolger et al. 2002; Casertano et al. 2003; Ferguson et al. 2003; Dahlen et al. 2003a). A complete listing of the GOODS SNe and their classifications can be found in Strolger et al. (2003). Here we focus on the initial identification via the apparent UV deficit of 15 candidate SNe Ia in three redshift intervals: $z<1.0,1<z<1.5$, and $z>1.5$.

\section{High, Higher, Highest}

For four SNe Ia with $0.2<z<1$, the apparent magnitude of $21<f 850 l p<23.5$ mag made it possible to get a spectroscopic confirmation in a few hours on a $6 \mathrm{~m}$ to $10 \mathrm{~m}$ telescope (three others were confirmed in ACS grism images). Each of these spectra provide conclusive identification as a SN Ia, as well as the indicated redshift, and are presented elsewhere (Strolger et al. 2003; Riess et al. 2003). In all cases the measured photometric redshifts and colors were consistent with the spectroscopic identification as shown in Figure 2. However, because the effective rest-frame wavelength of the $f 606 \mathrm{w}$ bandpass remains redward of $3300 \AA$ at $z<1$, the observed colors do not sample the UV deficit and were therefore insufficient to distinguish the transients as probable SNe Ia from colors alone.

For five transients, the observed colors and the photometric redshifts of the hosts were consistent with being SNe Ia at $1<z<1.5$ and inconsistent with being SNe II. In this redshift interval the observed colors were highly indicative of SN type, allowing us to select these as likely SNe Ia for target of opportunity (ToO) monitoring with HST in advance

of spectral confirmation and without delays or gaps in the photometric record. Four of 
the five were spectroscopically confirmed as SNe Ia at a redshift consistent with the initial photometric estimate as shown in Figure 2 (Riess et al. 2003).

The deep imaging obtained by GOODS before the discovery of SN $2002 \mathrm{fw}$ indicated $1.1<z<1.5$ with $95 \%$ confidence for its host galaxy. The measured colors of SN $2002 \mathrm{fw}$ at discovery of $f 775 w-f 850 \mathrm{lp}=0.80 \pm 0.05 \mathrm{mag}$ and $f 606 \mathrm{w}-f 850 \mathrm{lp}=3.0 \pm 0.1 \mathrm{mag}$ were consistent with a SN Ia at the photometric redshift (but not a SN II), precipitating a prompt HST monitoring (ToO). In Figure 3 we present the HST spectroscopy of SN $2002 \mathrm{fw}$, a SN Ia at $z=1.3$ and the first high-redshift SN Ia harvested from the GOODS program (see $\S 3$ ). The spectrum was obtained in $15 \mathrm{ks}$ of exposure with the ACS WFC and the grism filter, beginning 10 days after the discovery image. Similar ACS grism data was obtained for other SNe Ia in this redshift range and will be shown elsewhere (Riess et al. 2003).

Two SNe were discovered whose photometric redshifts and colors were indicative of SNe Ia at $z>1.5$. In both cases the $f 775 w-f 850 l p$ color was between 2.0 and $2.5 \mathrm{mag}$, consistent with the UV deficit of SNe Ia and strongly inconsistent with the bluer colors

of SNe II. The absence of either SN in the $f 606 \mathrm{w}$ images to the detection limit of point sources (with known position) limits $f 606 w-f 850 l p>3.2$ mag for each, consistent with SNe Ia and inconsistent with SNe II. Elsewhere we present the follow-up observations of these SNe and provide further constraints on their type and distance (Riess et al. 2003).

\section{Discussion}

The UV deficit is a useful tool to identify likely SNe Ia for rapid follow-up observations. For the GOODS survey careful consideration was given to select passbands which could use this diagnostic to discriminate SNe I from SNe II at $z>1$. Monte Carlo simulations of the selection of SNe Ia at $z>1$ indicate that the frequency of "false positives" (i.e., non-SNe Ia or SNe Ia at $z<1$ ) would be $<10 \%$ for expected transients. Further, in each actual case, we can calculate the likelihood of a misidentification taking into account the photometric measurements, their uncertainties, and the quality of the fit. The expectedly high level of success in identifying SNe Ia at $z>1$ allowed us to select suitable candidates and commit to rapid follow-up with $H S T$ in advance of any additional confirmation. The subsequent spectroscopy confirmed statistically and on an individual basis the good likelihood of selecting SNe Ia via their UV deficit.

A few SNe were discovered at $z>0.7$ whose blue color could be used to reject a classification of SN Ia, and matches well with classification as a young SN II (e.g., 
$f 606 w$ - $f 850 l p=0.0$ to $1.0 \mathrm{mag}$ and $z=0.9$; see figure 2 ). However the signal-to-noise ratio required to confirm spectroscopically the photometric evidence for young candidate $\mathrm{SNe}$ II at these redshifts far exceeds that of SNe Ia due both to weakness of features and the candidate's relative faintness and we did not get enough data to confirm this classification.

Due to its high redshift, the well-measured part of the spectrum of SN $2002 \mathrm{fw}$ extends from $2500 \AA$ to $4200 \AA$ (see Figure 3). For comparison we have superimposed the spectrum of the prototypical SN Ia 1981B at maximum extended to the UV by IUE (Branch et al. 1985). Overall the agreement between the two is excellent both in color and for specific features. Only minor differences are apparent, consistent with the intrinsic variation of SN Ia abundances and explosion velocities. Notable and pronounced absorption features in the spectrum of SN $2002 \mathrm{fw}$ include the Ca II H\&K feature at rest-frame $3700 \AA$ and the blueshifted Si II $\lambda 4130$. In the UV region of the spectrum, a rise blueward of $3200 \AA$ is well discerned due to blending of high-velocity Fe II absorption. Our line identifications are from Kirshner et al. (1993), and Mazzali et al. (1993). The defining feature of SNe Ia, Si II $\lambda 6355$ (typically blueshifted to $6150 \AA$ ) cannot be discerned due to the high redshift of the SN. However, as discussed by Coil et al. (2000) for SN $2002 \mathrm{fv}(z=1.2)$, the absorption feature near $4000 \AA$ due to Si II $\lambda 4130$ is sufficient to establish SN $2002 \mathrm{fw}$ as a SN Ia (though not as useful at later times as Si II $\lambda 6355$ ), and not a SN Ib/Ic supernova.

Light curves are a critical component of measuring distances to SNe Ia. They constrain the age of SN Ia observations, an individual calibration of each supernova's luminosity, and the extinction of each SN Ia (via the color excess), a prerequisite for calibrating their distances. For $z>1$ objects, this requires observations in the near-IR with NICMOS. Elsewhere (Riess et al. 2003), we present the distances measured for the GOODS SNe Ia based on their light curves and colors, as well as the implied cosmological constraints.

We thank resident and visiting supernova searchers including Tomas Dahlen, Ray Lucas, Norman Grogin, Peter Garnavich, Ann Hornshmeier, Lexi Moustakas, Marin Richardson, Ned Taylor, Claudia Kretchmer, Rafal Idzi, Kyoungsoo Lee, Richard Hook, Vicki Laidler, Carl Biagetti, Duilia de Mello, Swara Ravindranath, and Brian Schmidt. We also thank the steady and heroic efforts by OPUS, Dorothy Fraquelli, William Januszewski, Mark Calvin, Mark Kochte, Tracy Ellis, and Bill Workman.

Financial support for this work was provided by NASA through programs GO-9352 and GO-9583 from the Space Telescope Science Institute, which is operated by AURA, Inc., under NASA contract NAS 5-26555. Some of the results presented herein were made possible by the generous financial support of the W. M. Keck Foundation. Aldering, G., et al. 1998, IAU Circ. 7046 
Baron, E., et al. 2000, ApJ, 545, 444

Benítez, N., Riess, A., Nugent, P., Dickinson, M., Chornock, R., \& Filippenko, A. V. 2002, ApJ, 577, L1

Blakeslee, et al. 2003, ApJ, in press (astro-ph/0302402)

Branch, D., Doggett, J. B., Nomoto, K., \& Thielemann, F.-K. 1985, ApJ, 294, 619

Cappellaro, E., Evans, R., \& Turatto, M. 1999, A\&A, 351, 459

Cappellaro, E., Turatto, M., Tsvetkov, D. Yu., Bartunov, O. S., Pollas, C., Evans, R., \& Hamuy, M. 1997, A\&A, 322, 431

Casertano, S., et al. 2003, IAU Circ. 8052

Clocchiatti, A., et al. 2000, ApJ, 529, 661

Coil, A. L., et al. 2000, ApJ, 544, L111

Dahlen, T., et al. 2003a, IAU Circ. 8081

Dahlen, T., et al. 2003c, in preparation

Ferguson, H. C., et al. 2003, IAU Circ. 8069

Filippenko, A. V. 1997, ARAA, 35, 309

Filippenko, A. V., Li, W. D., Treffers, R. R., \& Modjaz, M. 2001, in Small-Telescope Astronomy on Global Scales, ed. W. P. Chen, C. Lemme, \& B. Paczyński (San

Francisco: ASP), 121

Ford, H., et al. 2003 (ACS paper)

Germany, L. M., et al. 2003, A\&Ap, in press

Giavalisco, M., et al. 2002, IAU Circ. 7981

Giavalisco, M., et al. 2003 (GOODS paper)

Gilliland, R. L., \& Phillips, M. M. 1998, IAU Circ. 6810

Hamuy, M., Phillips, M. M., Maza, J., Suntzeff, N. B., Schommer, R. A., \& Aviles, R. 1996, AJ, 112, 2391

Kirshner, R. P., et al. 1993, ApJ, 415, 589

Leibundgut, B. 2001, ARAA, 39, 67

Leibundgut, B., et al. 2003, in preparation

Lentz et al., 2001, ApJ, 547, 406

Li, W., Filippenko, A. V., Treffers, R. R., Riess, A. G., Hu, J., \& Qiu, Y. 2001, ApJ, 546, 734

Mazzali, P. A., Lucy, L. B., Danziger, I. J., Gouiffes, C., Cappellaro, E., \& Turatto, M. 1993, A\&Ap, 269, 423

Pain, R., et al. 1996, ApJ, 473, 356

Perlmutter, S., et al. 1999, ApJ, 517, 565

Poznanski, D., Gal-Yam, A., Maoz, D., Filippenko, A. V., Leonard, D. C., \& Matheson, T. 2002, PASP, 114, 833

Richardson, M., et al. 2002, xxxxx 
Riess, A. G. 2000, PASP, 112, 1284

Riess, A. G., et al. 1998, AJ, 116, 1009

Riess, A. G., et al. 2001, ApJ, 560, 49

Riess, A. G., et al. 2002, IAU Circ. 8012

Riess, A. G., et al. 2003, in preparation

Schmidt, B. P., et al. 1998, ApJ, 507, 46

Strolger, L.-G., et al. 2002, IAU Circ. 8038

Strolger, L.-G., et al. 2003, in preparation

Suntzeff, N., et al. 2003, in preparation

Tonry, J., et al. 1999, IAU Circ. 7312

Tonry, J. L., et al. 2003, ApJ, submitted

Wheeler, J. C., \& Harkness, R. P. 1990, Rep. Mod. Phys. 53, 1467 
Figure Captions

Figure 1:Upper Panel: High-resolution spectra of three representative young SNe; SN Ia 1992A (Kirshner et al. 1993) and a blue SN II (SN 1998S; Lentz et al. 2001) and a redder SN II (SN 1999em; Baron et al. 2000) from $1200 \AA$ to $7000 \AA$ normalized in the optical. Lower Panel: low-resolution sampling of the same spectra showing the color differences in the UV. Fe II resonantly scatters much of the thermal continuum flux of SNe Ia in the UV $(\lambda<3300 \AA)$, imprinting a characteristic "UV deficit" not seen in the hydrogen-rich photospheres of common SNe II.

Figure 2: Finding and classifying SNe Ia with ACS via the UV deficit. The expected $i-z$ (i.e., $F 775 W-F 850 l p$ ) and $v-z$ (i.e., $F 606 W-F 850 l p$ ) colors of SNe Ia and SNe II near maximum brightness (calculated from the SEDs in figure 1) compared to the observed colors of candidate SNe Ia found in GOODs data. SNe Ia are readily distinguishable from SNe II by their red colors. Reddened SNe II would be too faint for this magnitude-limited sample. SNe shown at $z<1.5$ were independently confirmed to be SNe Ia.

Figure 3: Spectrum of SN Ia $2002 \mathrm{fw}$ at $z=1.3$ as observed in $15 \mathrm{ks}$ through the grism filter with ACS on HST compared to SN Ia 1981B at maximum light. Identifiable absorption features in the spectrum of SN $2002 \mathrm{fw}$ include the Ca II H\&K absorption blend at rest-frame $3700 \AA$ and the blueshifted Si II $\lambda 4130$ absorption. 


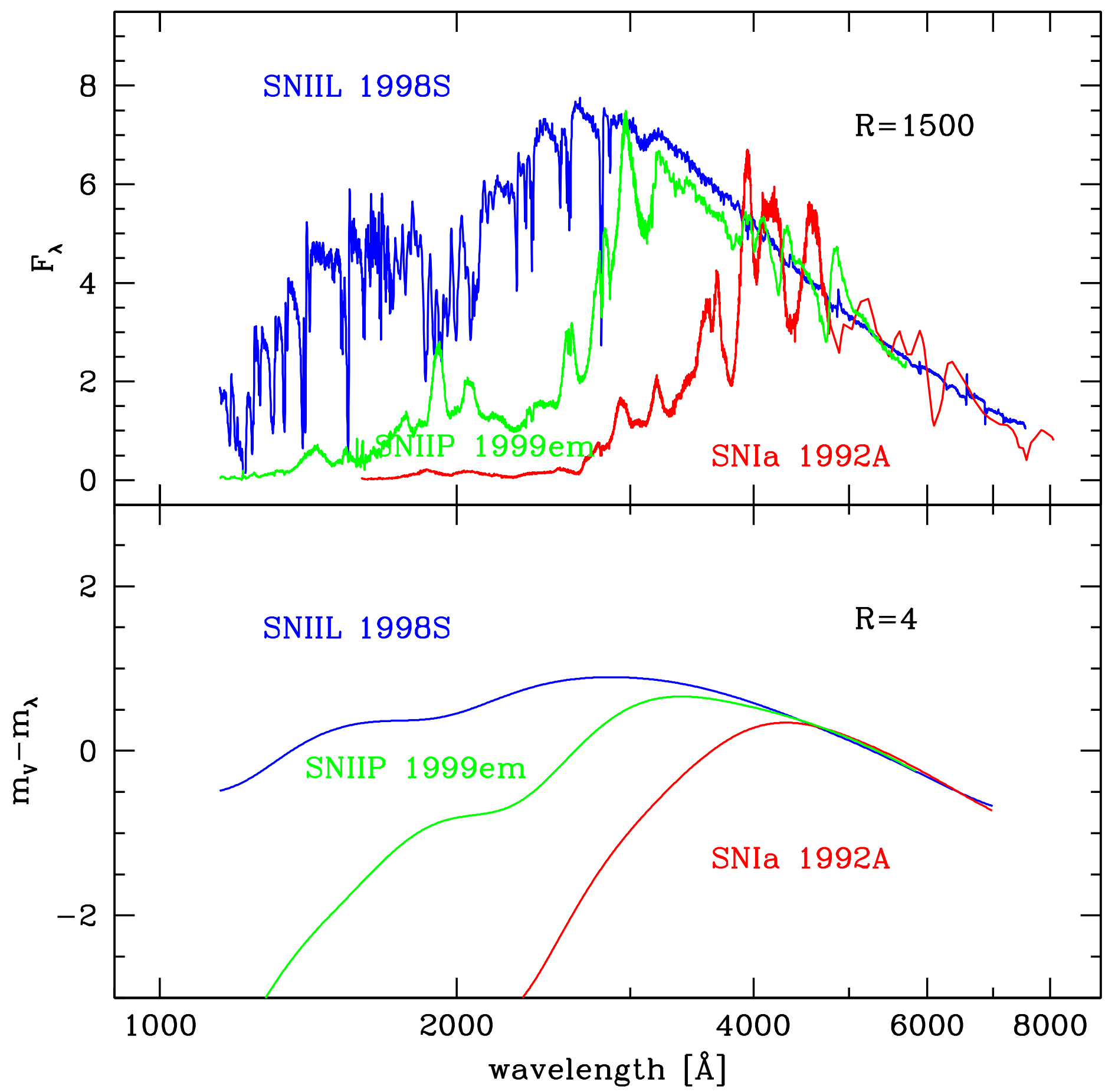




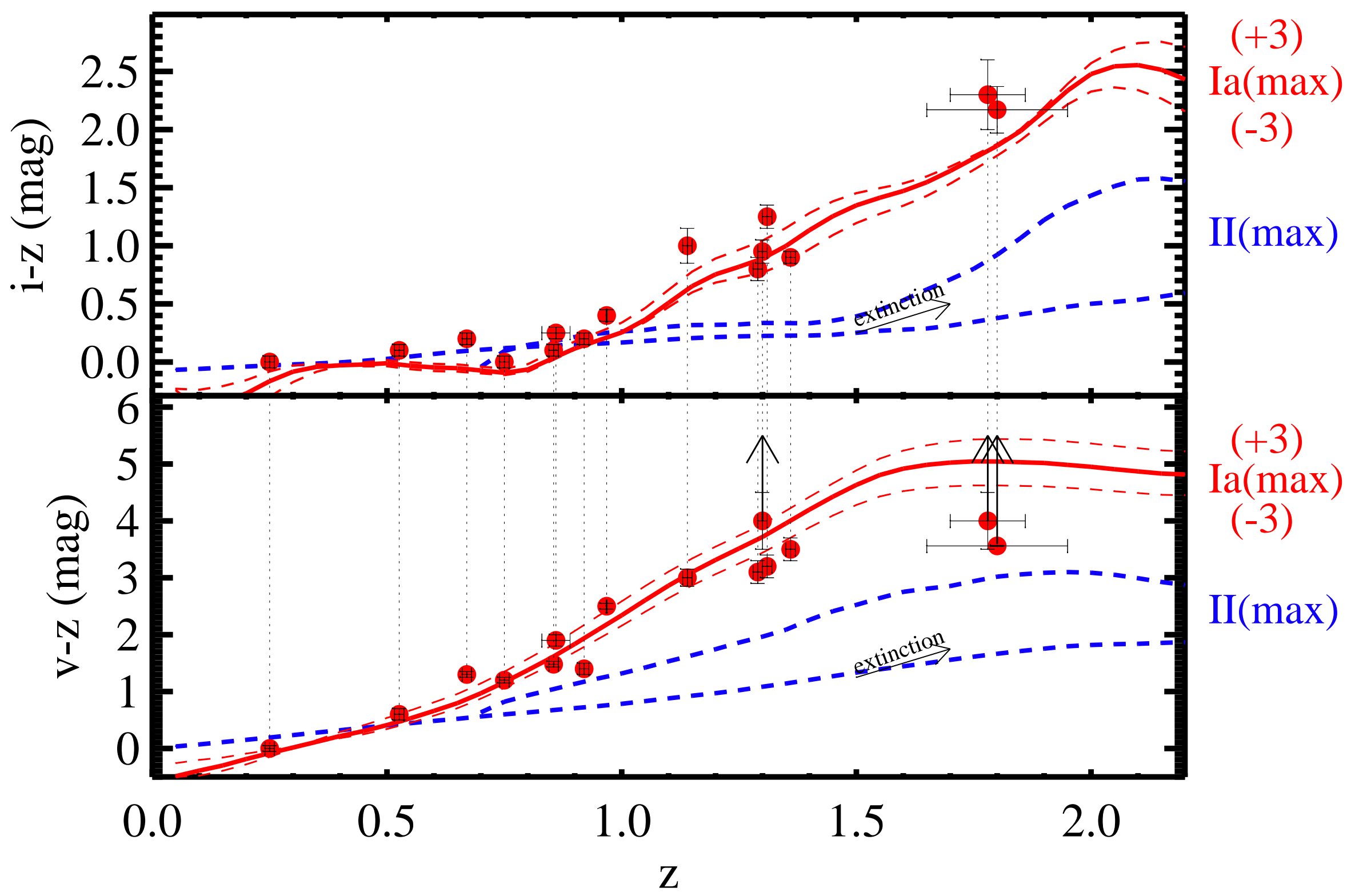




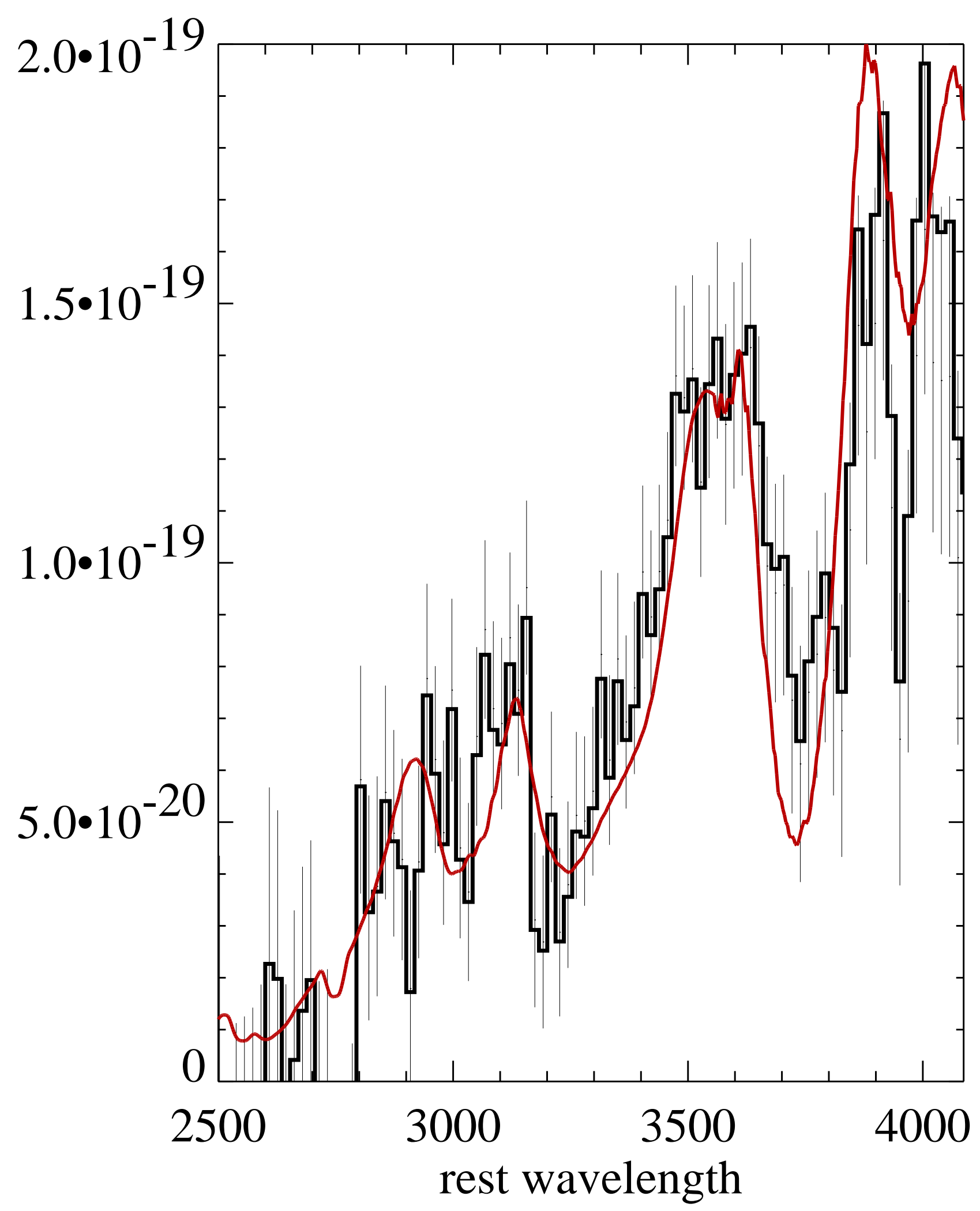

